\title{
Agalsidase beta treatment is associated with improved quality of life in patients with Fabry disease: Findings from the Fabry Registry
}

\author{
Torquil Watt, $M D^{1}$, Alessandro P. Burlina, $M D^{2}$, Chiara Cazzorla, PhD ${ }^{3}$, Dorothee Schönfeld, $M D^{4}$, \\ Maryam Banikazemi, MD ${ }^{5}$, Robert J. Hopkin, MD ${ }^{6}$, Ana Maria Martins, MD, PhD ${ }^{7}$, \\ Katherine Sims, $M D^{8}$, Dana Beitner-Johnson, PhD ${ }^{9}$, Fanny O'Brien, $P h D^{9}$, \\ and Ulla Feldt-Rasmussen, MD, DMSc ${ }^{l}$
}

\begin{abstract}
Purpose: To evaluate the effect of agalsidase beta on longitudinal healthrelated quality of life in patients with Fabry disease. Methods: The SF-36 ${ }^{\circledR}$ Health Survey was used to measure health-related quality of life in Fabry Registry patients. Seventy-one men and 59 women who were treated with agalsidase beta (median dose: $1.0 \mathrm{mg} / \mathrm{kg} / 2$ weeks) and who had baseline and at least 2 yearly posttreatment health-related quality of life measurements were included in these analyses. A repeated measures model was used to analyze change in score from baseline. Results: Men improved in the physical component summary and in all eight scales of the SF-36 after 1 and 2 years and in the mental component summary after 1 year of agalsidase beta treatment $(P<0.05)$. Women improved in the mental component summary and in six of the eight scales after 1 and/or 2 years of treatment. Patients whose baseline SF-36 scores were below the median showed the greatest improvements. These responses were comparable with or greater than the published effects of various treatments for multiple sclerosis, rheumatoid arthritis, central neuropathic pain, and Gaucher disease. Conclusion: Long-term treatment with agalsidase beta resulted in substantial improvements in health-related quality of life in both men and women; the effect was more pronounced in men. Genet Med 2010:12(11):703-712.
\end{abstract}

Key Words: lysosomal storage disease, alpha galactosidase A deficiency, SF-36 ${ }^{\circledR}$ Health Survey, enzyme replacement therapy, health status indicator

Eabry disease is a rare X-linked metabolic disorder caused by - insufficient activity of the lysosomal enzyme $\alpha$-galactosidase A. As a result, glycolipids with terminal $\alpha$-galactosidic

From the ${ }^{1}$ Department of Endocrinology, National University Hospital, Copenhagen, Denmark; ${ }^{2}$ Department of Neurology, San Bassano Hospital, Bassano del Grappa; ${ }^{3}$ Department of Neuroscience, Università degli Studi di Padova, Padua, Italy; ${ }^{4}$ Department of Medicine, University of Würzburg, Würzburg, Germany; ${ }^{5}$ Department of Pediatrics, Division of Clinical Genetics, Columbia University College of Physicians and Surgeons, New York City, New York; ${ }^{6}$ Division of Human Genetics, Cincinnati Children's Hospital, Cincinnati, Ohio; Inatos do Metabolismo-CREIM, Universidade Federal de São Paulo, São Paulo, Brazil; ${ }^{8}$ Department of Neurology, Massachusetts General Hospital, Boston; and ${ }^{9}$ Department of Biomedical Data Sciences and Informatics, Genzyme Corporation, Cambridge, Massachusetts.

Ulla Feldt-Rasmussen, MD, PhD, DMSc, National University Hospital, Blegdamsvej 9, DK-2100 Copenhagen, Denmark. E-mail: ufeldt@rh.dk.

Disclosure: The Fabry Registry is sponsored by Genzyme Corporation. U.F.-R., K.S., R.J.H., M.B., A.M., and A.P.B. serve on the Genzymesponsored Fabry Registry Boards of Advisors. D.B.-J. and F.O.B. are fulltime employees of Genzyme Corporation. T.W. has received unrestricted grants from Genzyme for other research projects.

Submitted for publication March 30, 2010

Accepted for publication June 19, 2010.

Published online ahead of print September 29, 2010.

DOI: 10.1097/GIM.0b013e3181f13a4a linkages, particularly globotriaosylceramide, accumulate in various tissues and cell types. ${ }^{1}$ The progressive accumulation of glycolipids eventually impairs critical organ function. The early symptoms of Fabry disease, including neuropathic pain in the extremities (acroparesthesia), hypohidrosis, and gastrointestinal discomfort typically begin during childhood. ${ }^{2-4}$ Some patients experience severe episodes of neuropathic pain (Fabry pain crises); these episodes can last for a day or more and may occur every few weeks or months. Later in life, many patients develop life-threatening disease manifestations, including chronic renal disease, cardiovascular disease, and strokes. ${ }^{5-9}$ Patients with Fabry disease, particularly men, have a substantially reduced life expectancy. ${ }^{10}$ Clinical depression is common in patients with Fabry disease, and the probability that patients with Fabry disease suffer from depression is strongly correlated with the degree that symptoms interfere with their everyday lives. ${ }^{11}$ Compared with the general US population, patients with untreated Fabry disease, particularly women, exhibit substantially reduced health-related quality of life (HRQL) with increasing age. , $^{, 12-14}$

Enzyme replacement therapy (ERT) with agalsidase beta (recombinant human $\alpha$-galactosidase A) clears globotriaosylceramide from vascular endothelial cells of various tissues. ${ }^{15,16}$ Long-term agalsidase beta treatment also stabilizes renal function ${ }^{17,18}$ and cardiac function. ${ }^{19}$ However, there is little information on the effects of ERT on patients' self-reported HRQL, in terms of physical, mental, and social well-being. 17,20,21 The purpose of the current analyses was to evaluate the effect of agalsidase beta on HRQL outcomes in Fabry Registry patients, and the influence of age and HRQL levels at the time treatment was initiated.

\section{MATERIALS AND METHODS}

\section{Study population}

The Fabry Registry is an ongoing, observational database that compiles clinical and investigative data on an international cohort of patients with Fabry disease. As of June 5, 2009, the Fabry Registry had enrolled 3182 patients, including 1587 men and 1592 women. All patients with Fabry disease are eligible to enroll in the Fabry Registry, regardless of age, gender, symptoms, or whether they are receiving ERT. Patient and physician participation is voluntary. Patients provide informed consent through local institutional review boards/Ethics Committees and may decline to participate or withdraw consent at any time.

SF-36 data were collected from Fabry Registry patients aged 14 years and older. The Fabry Registry recommends that participating physicians collect SF-36 data from their patients at the time of enrollment and every 6 months thereafter. ${ }^{22}$ Fabry Registry patients included in these analyses were required to 
have a baseline SF-36 assessment within 12 months before initiating agalsidase beta treatment. Men were required to have at least 3 yearly posttreatment SF-36 assessments (i.e., 0 to $<12$ months, 12 to $<24$ months, and 24 to $<36$ months). Too few women had 3 years of posttreatment SF-36 assessments available to perform meaningful statistical analyses at that time point; therefore, women were required to have at least 2 yearly posttreatment SF-36 assessments ( 0 to $<12$ months and 12 to $<24$ months).

\section{Outcome measure}

The SF-36 ${ }^{\circledR}$ Health Survey is a validated instrument that has been extensively used to evaluate HRQL in patients with various chronic diseases and in the general population. ${ }^{23,24}$ The SF-36 includes eight scales that relate to various self-reported aspects of physical, mental, and social well-being and function, and two summary measures: the physical component summary (PCS) score and mental component summary (MCS) score. The eight scales and the characteristics they measure include Physical Functioning, limitations in performing various physical activities; Role Physical, impact of physical health on completion of work or other daily activities; Bodily Pain, pain magnitude and its effect on daily activities; General Health, overall health perception; Vitality, perceived energy levels; Social Functioning, impact of physical and mental health on the ability to engage in normal social activities; Role Emotional, impact of emotional health on work or other daily activities; and Mental Health, self-reported mood levels. ${ }^{24}$ The scores for all scales are on a 100-point scale, with a higher score indicating better HRQL. In the general adult US population, average SF-36 scores range from 64 to 87 in men $(N=1055)$ and from 58 to 82 in women $(N=1412) .{ }^{23}$ Seven points has been reported to represent a clinically meaningful improvement in HRQL. ${ }^{25}$ For the Vitality subscale specifically, five points has been reported to represent a clinically meaningful improvement in groups with Vitality scores below average, ${ }^{26}$ as do patients with Fabry disease. ${ }^{8}$

\section{Clinical events}

The long-term progression of Fabry disease is associated with chronic renal disease, cardiovascular disease, and cerebrovascular events. Renal events were defined as chronic renal dialysis ( $>40$ days) or renal transplantation. Cerebrovascular events were defined as strokes. Cardiovascular events were defined as any arrhythmia (including atrial and ventricular), myocardial infarction, congestive heart failure, angina pectoris, or invasive cardiac interventions (e.g., pacemaker placement, coronary bypass, percutaneous coronary intervention valve replacement, and transplantation).

\section{Statistical analyses}

The effect of agalsidase beta treatment on HRQL was evaluated for each gender by calculating changes in score from baseline to the various time points for each of the eight SF-36 scales in a repeated measures model using SAS statistical software 9.1 (SAS Institute Inc., Cary, NC). Age at baseline was included as a covariate in the repeated measure models for the analyses of all patients but not for the analyses of patients subgrouped by age or by baseline SF-36 scores. Differences were considered to be statistically significant if $P<0.05$ in the repeated measures analyses. To further evaluate the effect of age at initiation of treatment, patients were subgrouped into younger and older categories, within each gender, based on the median ages at the time treatment was started. The effect of baseline disease severity, in terms of impaired HRQL, was evaluated by subgroup analyses of patients with SF-36 scores below median for each scale, within each gender. Changes in SF-36 scores observed in agalsidase-beta treated patients were also qualitatively compared with changes in SF-36 scores reported in patients treated for other clinical disorders resembling Fabry disease.

\section{RESULTS}

As of June 5, 2009, 71 men in the Fabry Registry had SF-36 assessments reported at baseline and at least three posttreatment assessments during a period of 36 months. At that time, 59 women had SF-36 assessments reported at baseline and at least two posttreatment assessments during a period of 24 months. The 36-month data that were available for women $(N=28)$ generally resembled the 24-month data from the cohort of 59 women, across the various scales (data not shown). The 71 men had received agalsidase beta for an average period of $81 \pm 31$ months (mean \pm standard deviation) at $1.0 \pm 0.14 \mathrm{mg} / \mathrm{kg}$ every other week, as an averaged dose during the period of SF-36 data collection. The 59 women had received agalsidase beta for an average period of $41 \pm 22$ months (mean \pm standard deviation) at $0.9 \pm 0.22 \mathrm{mg} / \mathrm{kg}$ every other week, as an averaged dose during the period of SF-36 data collection.

Although the Fabry Registry recommends collecting SF-36 data every 6 months, ${ }^{22}$ a relatively small percentage of patients had longitudinal SF-36 data reported. The possibility that these analyses were susceptible to selection bias was evaluated by comparing the agalsidase beta-treated men and women included in these analyses with other agalsidase beta-treated men and women in the Fabry Registry (i.e., those for whom the necessary SF-36 assessments were not reported). These two groups of patients were compared separately within each gender by analyzing various demographic and clinical characteristics, as summarized in Table 1. The 71 men included in the HRQL analyses were diagnosed at a younger age than 898 other agalsidase beta-treated men in the Fabry Registry ( 24 vs. 30 years) and began treatment at a younger age (mean: 33 vs. 37 years). Compared with the 898 other agalsidase beta-treated men in the Fabry Registry, the 71 men included in the HRQL analyses had fewer renal events and fewer strokes before they initiated agalsidase beta treatment, as shown in Table 1. Among women, the only significant difference found between the two patient groups was that a higher proportion of the 59 women included in these analyses were from Europe (58\%), compared with the 404 other agalsidase beta-treated women in the Fabry Registry (40\%).

As shown in Table 2, men reported significantly higher PCS and MCS scores after 0 to $<12$ months of treatment. PCS scores were also significantly higher in men after 12 to $<24$ months of treatment. In women, MCS scores were significantly improved after 0 to $<12$ months and 12 to $<24$ months of agalsidase beta treatment. There was a trend toward higher PCS scores in women after treatment, but there may have been too few women in this cohort for the trend to reach statistical significance $(N=59)$.

As shown in Figure 1 (Panel A), men reported significantly higher scores in all eight scales of the SF-36 after 0 to $<12$ months and 12 to $<24$ months of agalsidase beta treatment. Men reported the greatest improvements in the Role Physical (9-15 points) and Role Emotional scales (6-11 points). After 24 to $<36$ months of treatment, significant improvements were observed in six of the eight scales (all except Social Functioning and Role Emotional). Mens' mean scores were slightly lower during the third year of treatment, compared with the first and 
Table 1 Demographic and clinical characteristics of Fabry Registry patients treated with agalsidase beta

Men in HRQL cohort Other Fabry Registry men Women in HRQL cohort Other Fabry Registry women $(N=71) \quad(N=898)$

Age at Fabry diagnosis ${ }^{a}(\mathrm{yr})$

$n$

Mean (SD)

Median (min, max)

Age at initiation of agalsidase beta treatment (yr)

Mean (SD)

Median (min, max)

Age at last follow-up (yr)

Mean (SD)

Median (min, max)

Geographic region, $n(\%)$

\section{Europe}

The United States

Clinical events ${ }^{d}$ before initiation of agalsidase beta

treatment, $n(\%)$

Renal events, $n(\%)$

Cardiovascular events, $n(\%)$

Strokes, $n(\%)$
$31(44)$

$30(42)$

10 (14)

$19(27)$

70

$24(13.9)^{b}$

$33(11.5)^{b}$

33 (16-66)

40 (11.1)

$40(23-71)$

41 (13.2)

$41(14-82)$

394 (43)

$340(38)$

164 (18)

$333(37)$

$5(7)^{c}$

150 (17)

213 (24)

$1(1)^{c}$
$(N=59)$

$(N=404)$

${ }^{a}$ Age at diagnosis was calculated based on the number of patients for whom diagnosis dates were available, as indicated.

${ }^{b} P<0.05$ by $t$ test.

${ }^{c} P<0.05$ by $\chi^{2}$ test (compared with other Fabry Registry patients of the same gender).

${ }^{d}$ As defined in section "Materials and Methods."

Table 2 Effect of agalsidase beta treatment on HRQL as measured by SF-36 PCS and MCS scores

\begin{tabular}{lcc}
\hline & $\begin{array}{c}\text { Men }(N=71), \\
\text { mean } \pm \text { SEM }\end{array}$ & $\begin{array}{c}\text { Women }(N=59), \\
\text { mean } \pm \text { SEM }\end{array}$ \\
\hline $\begin{array}{c}\text { Physical component } \\
\text { summary scores }\end{array}$ & & \\
$\quad$ Baseline & $38.8 \pm 1.29$ & $36.8 \pm 1.53$ \\
$>0-12$ mo & $42.1 \pm 1.19^{a}$ & $38.4 \pm 1.50$ \\
$>12-24$ mo & $43.0 \pm 1.30^{a}$ & $38.5 \pm 1.48$ \\
$>24-36$ mo & $41.3 \pm 1.29$ & - \\
Mental component & & \\
$\quad$ summary scores & & $45.9 \pm 1.41$ \\
Baseline & $46.0 \pm 1.22$ & $49.2 \pm 1.26^{a}$ \\
$>0-12$ mo & $48.9 \pm 1.08^{a}$ & $48.8 \pm 1.36^{a}$ \\
$>12-24$ mo & $48.4 \pm 1.20^{b}$ & - \\
$>24-36$ mo & $47.3 \pm 1.22$ & \\
\hline${ }^{a} P<0.05$ by repeated measures model using age at baseline as a covariate. \\
${ }^{b} P=0.0614$. \\
SEM, standard error of the mean.
\end{tabular}

second years, across all scales. However, there were no statistically significant differences between any of the scales at the 24 to $<36$-month time point versus the 0 to $<12$-month time point. Physical Functioning and Vitality scores were significantly lower at the 24 to $<36$-month time point compared with the 12 to $<24$-month time point, although mean scores remained significantly higher than pretreatment levels at that time.

Mean SF-36 scale scores for women are shown in Figure 1 (Panel B). Women reported significantly higher scores in six of the eight scales after 0 to $<12$ months and/or 12 to $<24$ months of agalsidase beta treatment (all except Physical Functioning and Role Emotional). Women reported the greatest improvements in the Role Physical (10 points) and General Health scales ( 9 points) after 0 to $<12$ months of treatment and in Social Functioning (10 points) after 12 to $<24$ months of treatment.

The influence of age at initiation of treatment on the effect of agalsidase beta treatment on HRQL is shown in Figure 2. Men who were older than the median age at the time they initiated treatment (33 years or older) reported substantial improvements in all scale scores at two or more time points (Panel A). These men reported the greatest improvements in the Role Physical (14-15 points) and the Role Emotional scales (20-22 points). The younger group of men reported significant gains in only the Role Physical and Bodily Pain scales (data not shown). Com- 


\section{A Men}

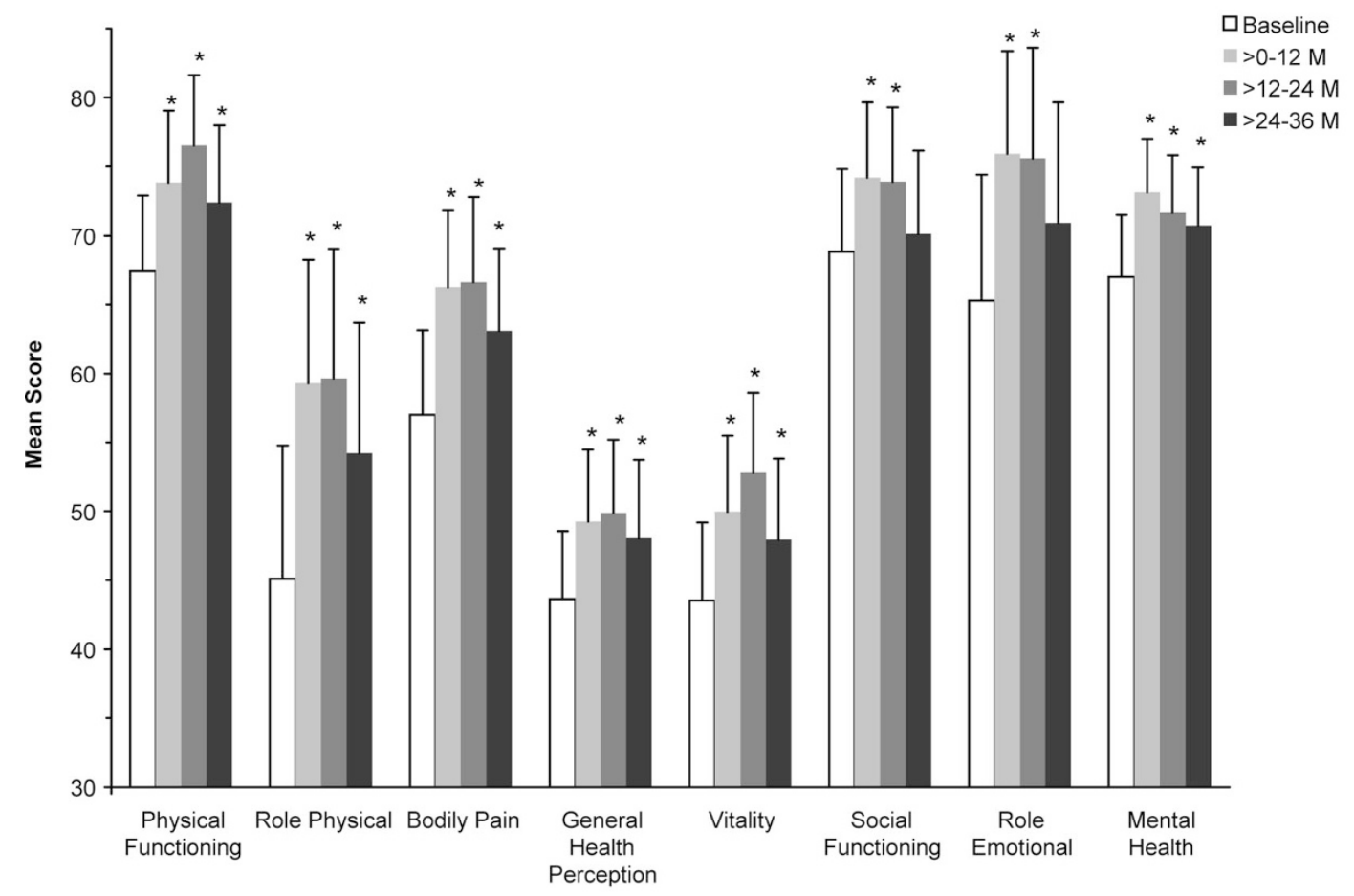

B Women

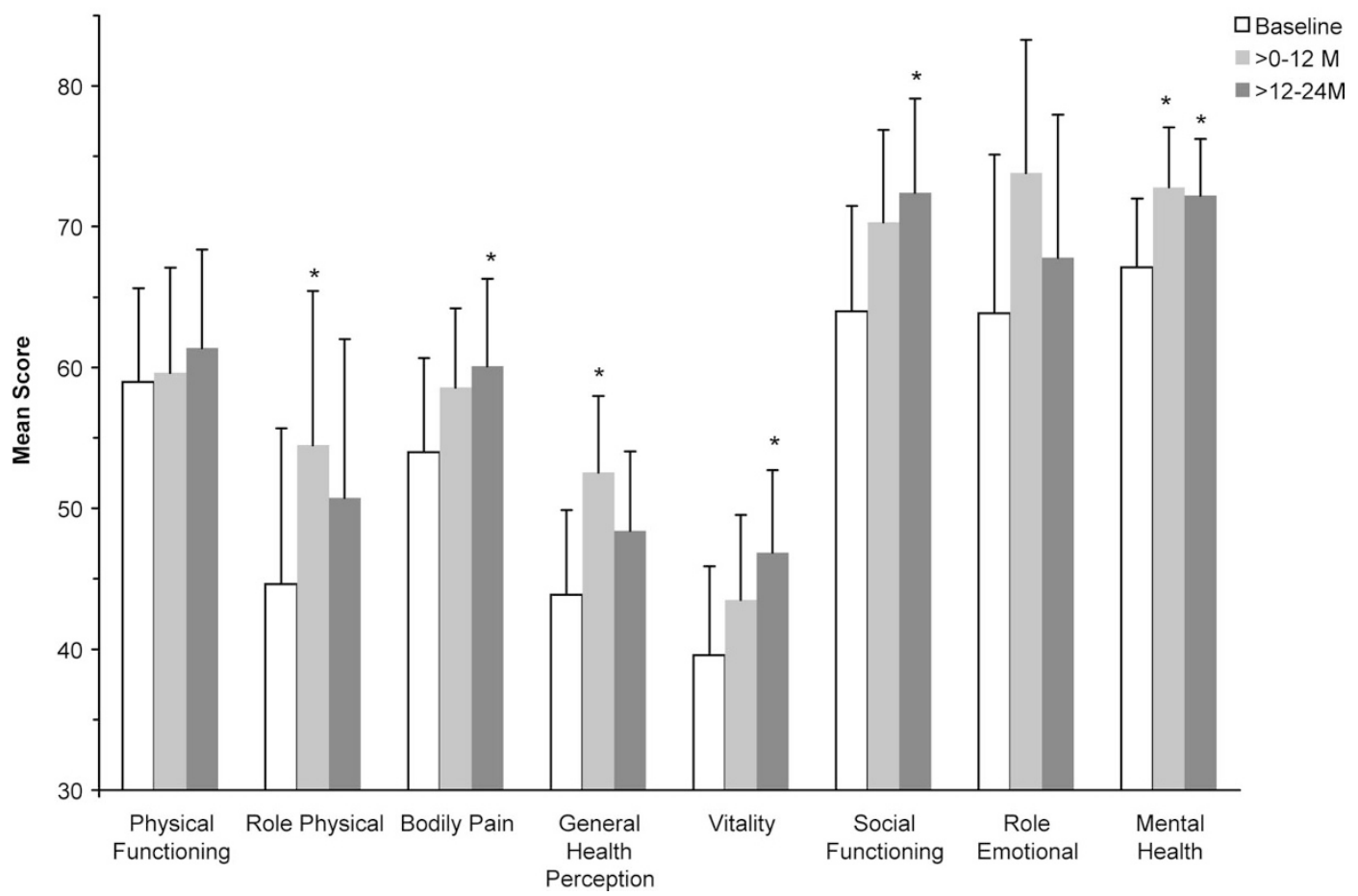

Fig. 1. Effect of agalsidase beta treatment on SF-36 scores in Fabry Registry men and women. Data are expressed as average SF-36 scores at baseline (open bars), after 0-12 months of treatment (light gray bars), 12-24 months of treatment (dark gray bars), and (for men only) 24-36 months of treatment (black bars). Error bars represent the upper limit of $95 \%$ confidence intervals. Panel A: average SF-36 scores among $N=71$ men. Panel B: average SF-36 scores among $N=59$ women. ${ }^{*} P<0.05$; significantly different from baseline value by repeated measures model, using age at baseline as a covariate. 


\section{A Men 33 years or older at treatment initiation}

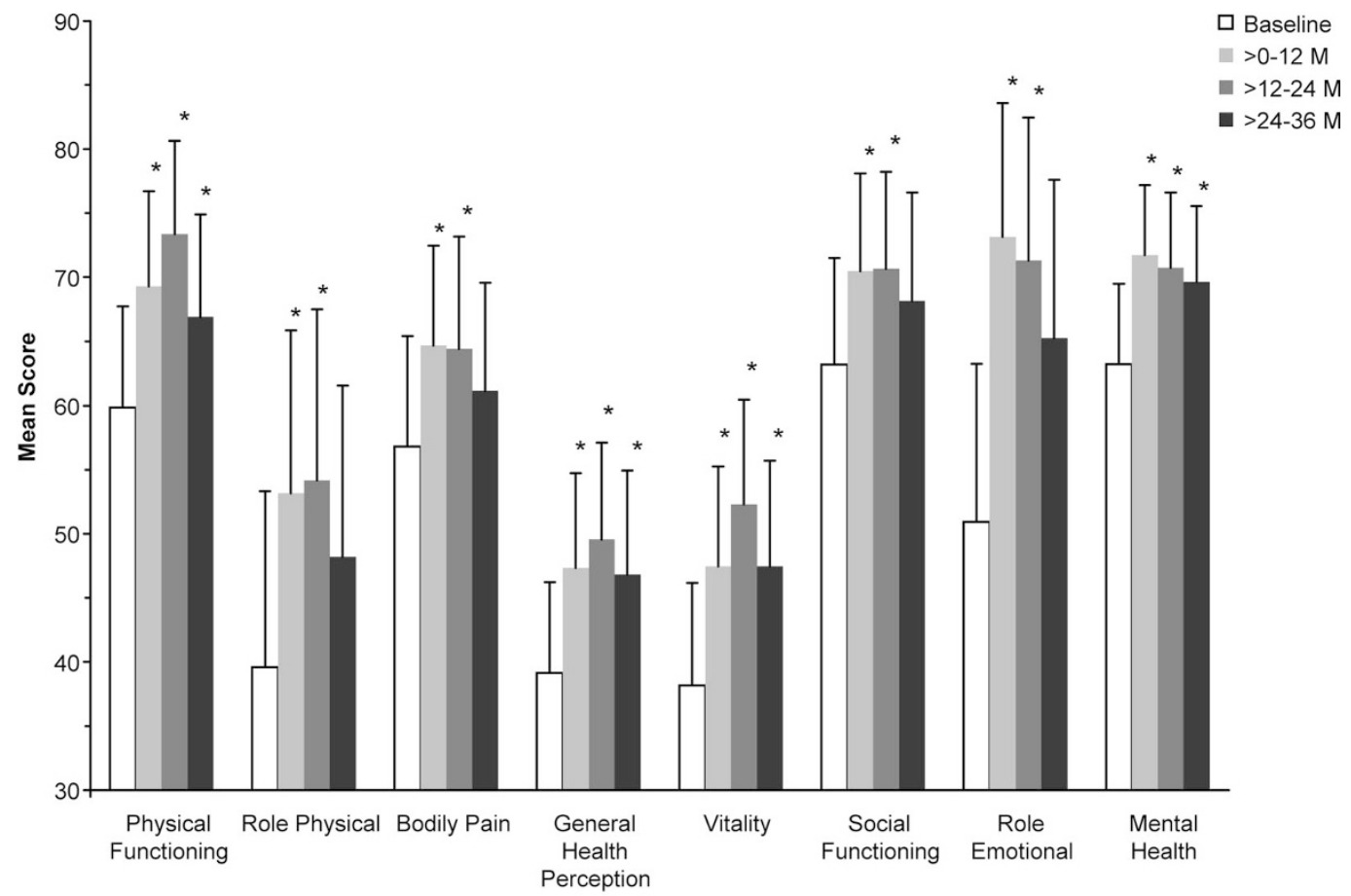

\section{B Women 48 years or older at treatment initiation}

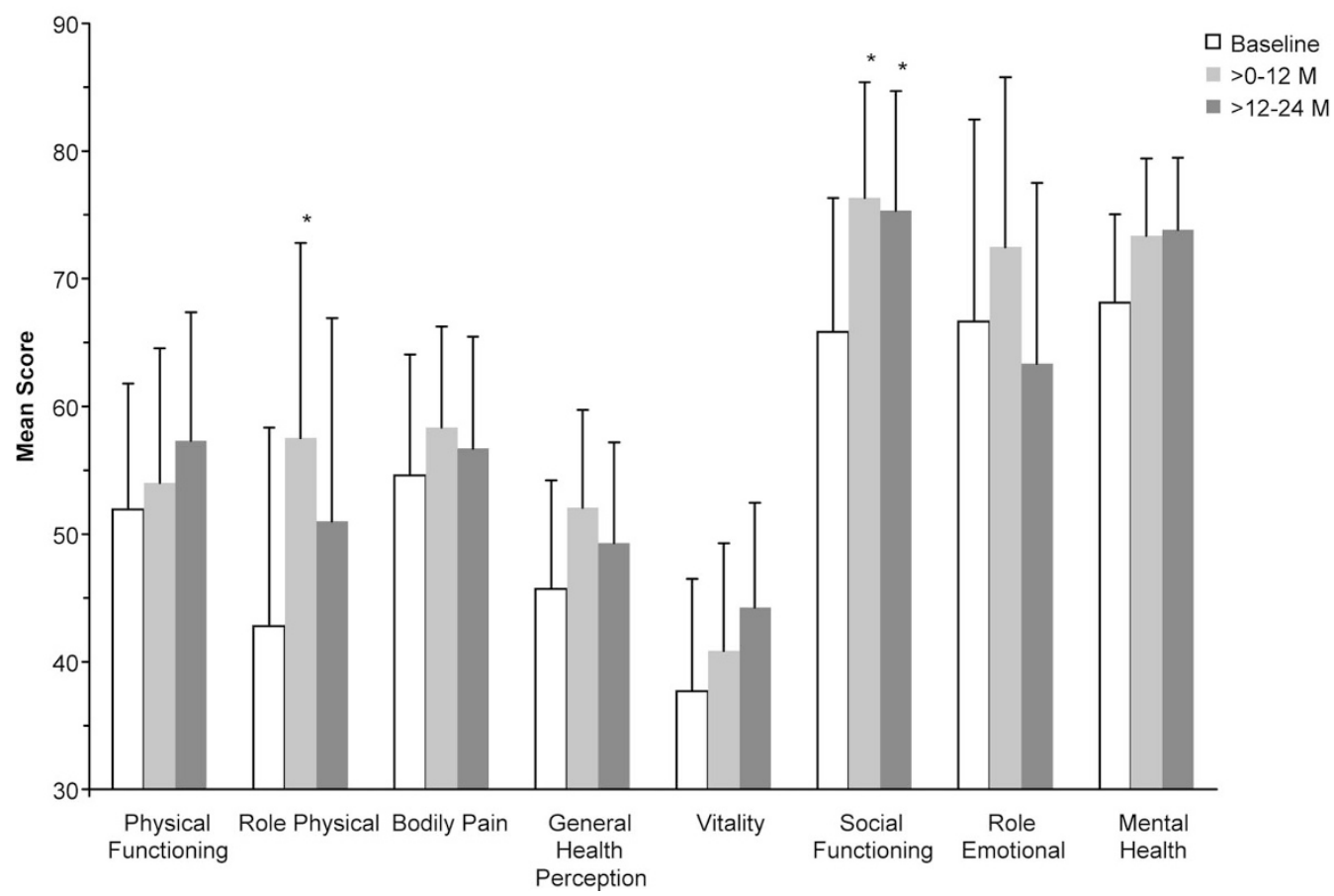

Fig. 2. Effect of agalsidase beta treatment on SF-36 scores among Fabry Registry patients older than median age at initiation of treatment. Data are expressed as average SF-36 scores at baseline (open bars), after 0-12 months of treatment (light gray bars), 12-24 months of treatment (dark gray bars), and (for men only) 24-36 months of treatment (black bars). Error bars represent the upper limit of $95 \%$ confidence intervals. Panel A: average SF-36 scores among $N=35$ men aged 33 years or older at the time agalsidase beta treatment was initiated. Panel B: average SF- 36 scores among $N=30$ women aged 48 years or older at the time agalsidase beta treatment was initiated. ${ }^{*} P<0.05$; significantly different from baseline value by repeated measures model. 


\section{A Men with scores below median at baseline}

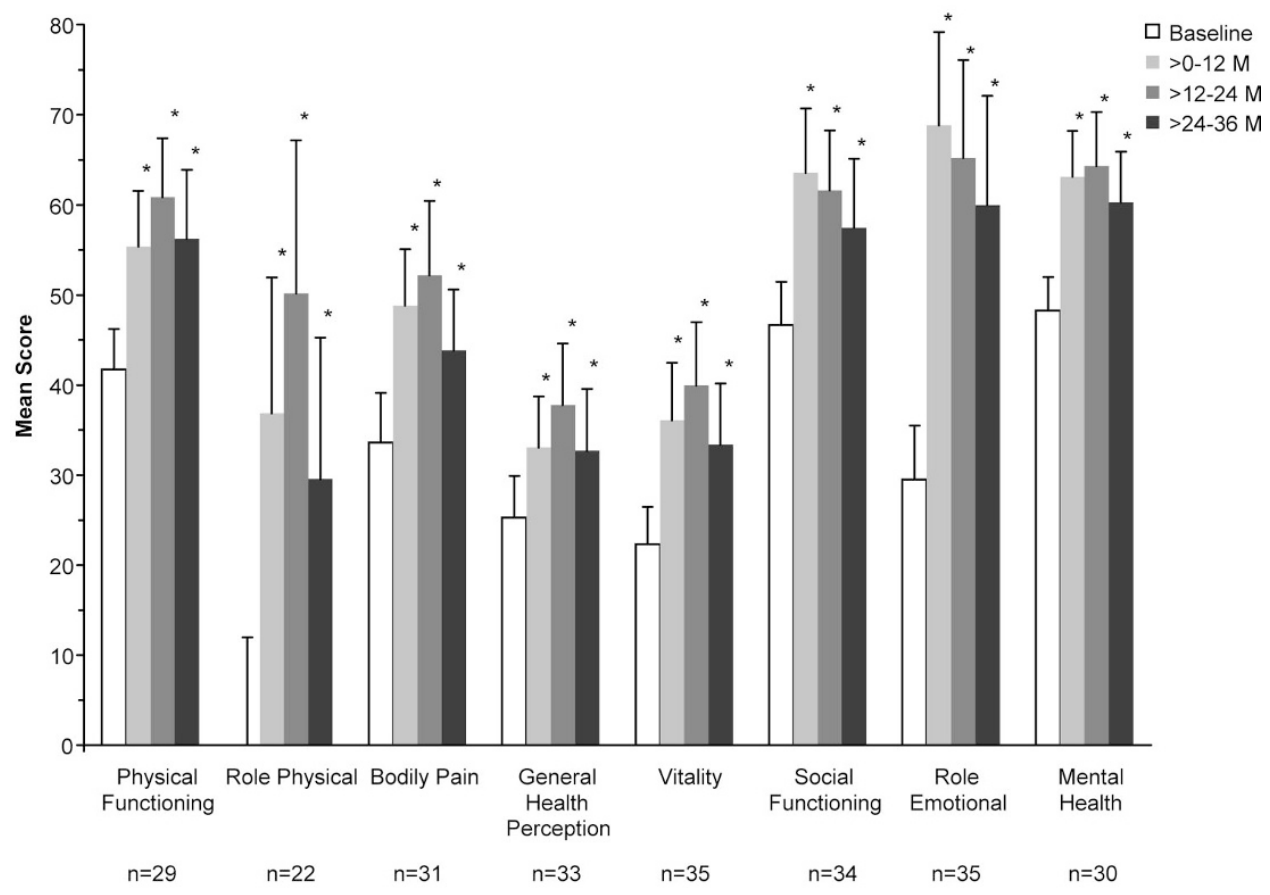

\section{B Women with scores below median at baseline}

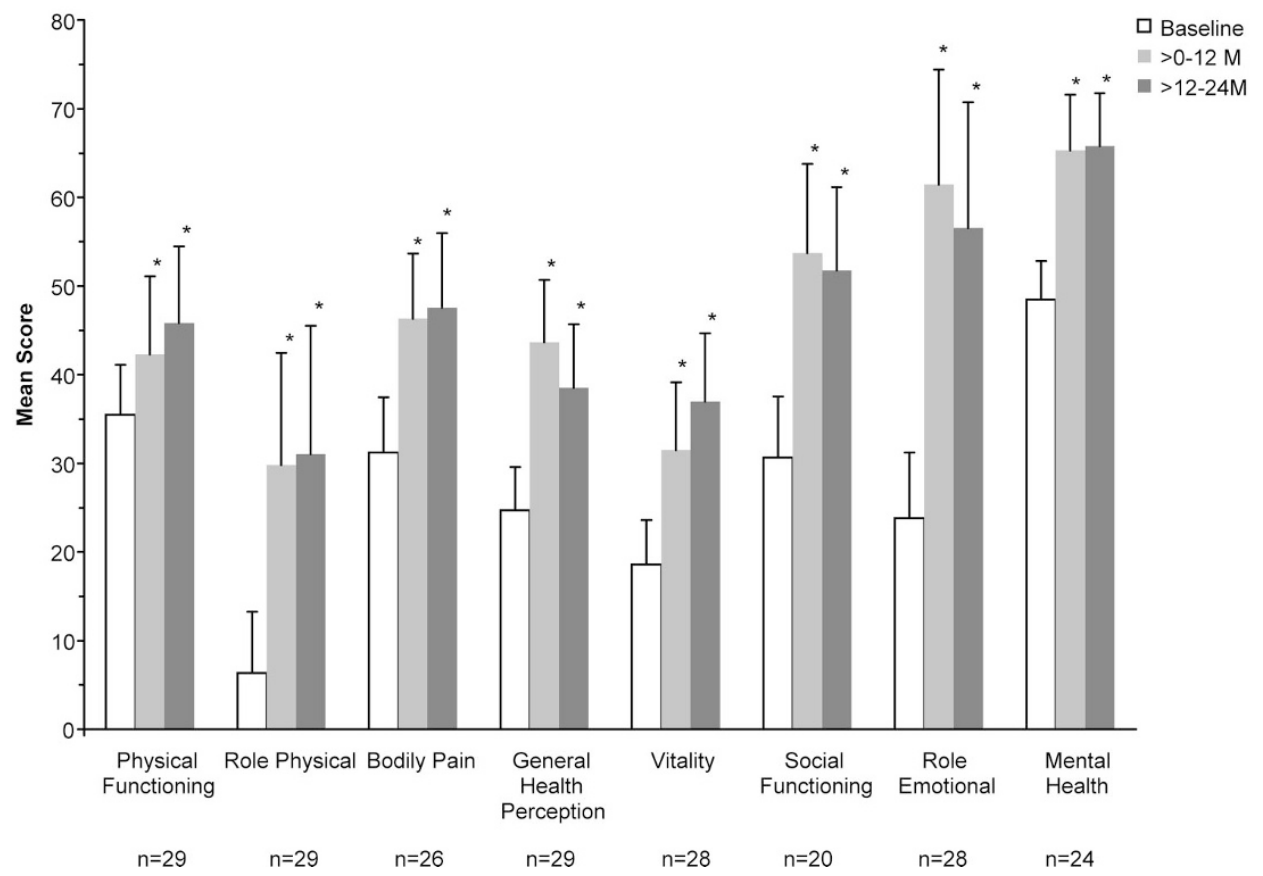

Fig. 3. Effect of agalsidase beta treatment on SF-36 scores among Fabry Registry patients with baseline scores below the median. Data are expressed as average SF-36 scores at baseline (open bars), after 0-12 months of treatment (light gray bars), 12-24 months of treatment (dark gray bars), and (for men only) 24-36 months of treatment (black bars). Error bars represent the upper limit of $95 \%$ confidence intervals. Panel A: average SF-36 scores among men with baseline scores less than the median. Panel B: average SF-36 scores among women with baseline scores below the median. The numbers of patients who had baseline scores less than the median are indicated below each scale on the $x$-axis. Different numbers of patients had scores below the median at baseline for each scale because some patients had baseline scores equal to the median for particular scales. ${ }^{*} P<0.05$; significantly different from baseline value by repeated measures model. 
pared with men who were younger than 33 years when they initiated treatment, the older men had significantly lower baseline SF-36 scores in the Physical Functioning and Role Emotional scales. The mean Physical Functioning score was 60 in older men versus 75 in younger men $(P=0.008)$, and the mean Role Emotional score was 51 in older men versus 80 in younger men $(P=0.002)$. Mean scores in the General Health, Vitality, Social Functioning, and Mental Health scales also tended to be lower in older men, but these differences (ranging from 8 to 11 points) did not achieve statistical significance ( $P$ values $>0.05-$ $0.10)$. In contrast, age at initiation of treatment was not a major factor in womens' overall response to agalsidase beta treatment, in terms of SF-36 scores. Women who were older than the median age at the time they initiated treatment (48 years or older) reported statistically significant gains in two scales: 15 points in Role Physical and 10-11 points in Social Functioning. Women who were younger than 48 years when they initiated treatment also reported significant gains in two scales: 10 points in Bodily Pain and 11 points in General Health (data not shown). Unlike in men, there were no consistent differences in baseline SF-36 scores between the older and younger groups of women (data not shown).

The effect of agalsidase beta treatment on HRQL in the subgroup of patients whose baseline scale scores were less than the median score of the overall cohort is shown in Figure 3. As shown in Panel A, men with baseline scores below the median reported large improvements in all eight SF-36 scales after 12, 24 , and 36 months of treatment. These patients reported the greatest improvements in the Role Physical (30-50 points) and Role Emotional scales (30-39 points). Similar results were reported by women whose baseline scores were less than the median score for each scale (Panel B). These women reported statistically significant improvements in all scales after up to 12 months and 24 months of agalsidase beta treatment. The greatest improvements of women reported were in the Role Physical (24-25 points), Social Functioning (21-23 points), and Role Emotional Scales (33-38 points).

Pain, a common symptom of Fabry disease, is an important part of HRQL, and these analyses indicate that Bodily Pain improves after treatment in both men and women. Beyond the SF-36, the Fabry Registry also asks physicians to indicate on case report forms whether their patients reported pain, the frequency of such pain, and what, if any, medications were used to treat pain. Unfortunately, only a subset of the patients in this cohort had this type of data recorded before the initiation of agalsidase beta treatment (20/71 men, $28 \%$ and 38/59 women, $64 \%$ ). The limited data available were insufficient to determine whether meaningful changes in the reported frequency of pain or in the use of pain medications occurred after treatment (data not shown).

The magnitude of improvement in HRQL of patients with Fabry disease after agalsidase beta treatment is shown in comparison with various treatments for other chronic diseases in Table 3. The upper half of Table 3 shows average baseline (pretreatment) SF-36 scale scores for Fabry Registry patients and scores previously reported for patients with rheumatoid arthritis, ${ }^{27}$ multiple sclerosis, ${ }^{28}$ central neuropathic pain, ${ }^{29}$ and Gaucher disease. ${ }^{30}$

Across most scales, Fabry Registry patients reported baseline SF-36 scores that were generally similar to those of patients with multiple sclerosis ${ }^{28}$ and patients with Gaucher disease. ${ }^{30}$ However, in terms of baseline General Health and Vitality scale scores, patients with Fabry disease had lower scores, more closely resembling patients with rheumatoid arthritis ${ }^{27}$ and central neuropathic pain. ${ }^{29}$ The lower half of Table 3 shows mean change from baseline in response to treatment with agalsidase beta (patients with Fabry disease), abatacept (patients with rheumatoid arthritis), natalizumab (patients with multiple sclerosis), pregabalin (patients with central neuropathic pain), and imiglucerase (patients with Gaucher disease). Across nearly all scales, the changes from baseline in response to agalsidase beta treatment reported by Fabry Registry men were substantially larger than the change from baseline in patients with rheumatoid arthritis treated with abatacept, in patients with multiple sclerosis treated with natalizumab, and patients with central neuropathic pain treated with pregabalin. There were two exceptions: the Mental Health scale improved by 4.6 points in response to agalsidase beta treatment in Fabry men, which was the same as the response to abatacept in rheumatoid arthritis; also, the Bodily Pain scale improved by 14.6 points in response to agalsidase beta treatment in Fabry men versus 15.6 points in response to patients with central neuropathic pain treated with pregabalin. The posttreatment improvements in Fabry mens' HRQL most closely resembled that of patients with Gaucher disease treated with imiglucerase for 24 months (Table 3 ).

\section{DISCUSSION}

These analyses were undertaken to evaluate the effect of agalsidase beta on HRQL. For this purpose, repeated measures analyses were used to evaluate longitudinal SF-36 data from 130 Fabry Registry patients who were treated with agalsidase beta for a minimum of 2 years.

In men, HRQL was improved after the first 2 years of treatment with agalsidase beta in all eight SF-36 scales and the PCS score. The greatest improvements were reported in the scales that measure the impact of physical and mental health on the ability to perform work and daily activities, i.e., the Role Physical and Role Emotional scales, respectively; these are core aspects of HRQL. The magnitude of these improvements in SF-36 scores is consistent with what has been reported to be clinically important. $25,26,31$ Men reported sustained improvements during the third year of treatment, except for the Social Functioning and Role Emotional scales. Mean SF-36 scores in men tended to be lower during the third year of treatment compared with the first year, although there was no statistically significant difference. Although a true decline in HRQL at longer follow-up periods cannot be excluded, it could be speculated that this tendency toward lower scores during the third year may reflect a "response shift," where patients' expectations are set at a higher level after an initial good response. ${ }^{32}$ Alternatively, this may represent a "ceiling effect," i.e., after initial improvements during the first year, further changes may be more difficult to appreciate. In addition, the SF-36 questionnaire asks about patients' well-being "in the last 4 weeks" and not "since baseline."

Women reported statistically significant improvements in six of the eight scales during the first 2 years of agalsidase beta treatment (all except Physical Functioning and Role Emotional) and the MCS score. The greatest improvements reported by women were in the Role Physical and General Health scales.

The summary component scores (e.g., PCS and MCS) suggest that men tended to experience more improvement in physical aspects of HRQL, whereas women tended to experience more improvement in mental or psychosocial aspects of HRQL. Overall, men experienced greater improvements in HRQL in response to agalsidase beta treatment, compared with women. This is probably related to the fact that men generally experience more severe manifestations of this X-linked disorder ${ }^{1,8}$ and, therefore, have more room for improvement in response to 
Table 3 Comparison of effects of various treatments on health-related quality of life in other chronic diseases

\begin{tabular}{|c|c|c|c|c|c|c|}
\hline & $\begin{array}{c}\text { Fabry disease } \\
\operatorname{men}^{a}(N=71), \\
\text { agalsidase } \\
\text { beta }(24 \mathrm{mo})\end{array}$ & $\begin{array}{c}\text { Fabry disease } \\
\text { women }^{a}(N=59), \\
\text { agalsidase } \\
\text { beta }(24 \mathrm{mo})\end{array}$ & $\begin{array}{c}\text { Rheumatoid } \\
\text { arthritis } \\
(N=258), 27 \\
\text { abatacept }(6 \mathrm{mo})\end{array}$ & $\begin{array}{c}\text { Multiple sclerosis } \\
(N=627), 28 \\
\text { natalizumab }(24 \mathrm{mo})\end{array}$ & $\begin{array}{l}\text { Central neuropathic } \\
\text { pain }(N=20),{ }^{29} \\
\text { pregabalin }(4 \mathrm{wk})\end{array}$ & $\begin{array}{l}\text { Gaucher disease } \\
(N=32),{ }^{30} \\
\text { imiglucerase } \\
(24 \mathrm{mo})\end{array}$ \\
\hline \multicolumn{7}{|l|}{ Mean baseline score } \\
\hline Physical functioning & $67.5(2.72)$ & $59.0(3.32)$ & 26.2 & 70.2 & 31.5 & 73.6 \\
\hline Role physical & $45.1(4.87)$ & $44.6(5.51)$ & 30.5 & 55.0 & 12.5 & 62.5 \\
\hline Bodily pain & $57.0(3.08)$ & $54.0(3.34)$ & 30.6 & 73.0 & 30.7 & 68.3 \\
\hline General health & $43.6(2.48)$ & $43.9(3.00)$ & 34.9 & 53.7 & 46.2 & 65.7 \\
\hline Vitality & $43.5(2.84)$ & $39.6(3.15)$ & 35.1 & 48.3 & 45.8 & 47.0 \\
\hline Social functioning & $68.8(3.00)$ & $64.0(3.74)$ & 33.0 & 71.4 & 51.3 & 73.4 \\
\hline Role emotional & $65.3(4.59)$ & $63.8(5.63)$ & 35.7 & 66.9 & 60.0 & 71.9 \\
\hline Mental health & $67.0(2.27)$ & $67.1(2.44)$ & 40.5 & 67.0 & 67.5 & 70.1 \\
\hline \multicolumn{7}{|l|}{$\begin{array}{l}\text { Mean change from } \\
\text { baseline score } \\
\text { after treatment }\end{array}$} \\
\hline Physical functioning & $9.1(2.31)^{b}$ & $2.4(2.66)$ & $5.3^{b}$ & $1.2^{b}$ & 2.5 & $9.0^{b}$ \\
\hline Role physical & $14.6(5.16)^{b}$ & $6.1(5.84)$ & $6.7^{b}$ & $6.8^{b}$ & 0 & $20.0^{b}$ \\
\hline Bodily pain & $9.6(2.75)^{b}$ & $6.1(3.02)^{b}$ & $8.7^{b}$ & -1.0 & $15.6^{b}$ & $9.6^{b}$ \\
\hline General health & $6.2(2.40)^{b}$ & $4.5(2.40)$ & $3.9^{b}$ & $3.8^{b}$ & 3.7 & 6.3 \\
\hline Vitality & $9.3(2.29)^{b}$ & $7.3(3.18)^{b}$ & $6.9^{b}$ & $3.7^{b}$ & 4.5 & $14.6^{b}$ \\
\hline Social functioning & $5.1(2.59)^{b}$ & $8.4(3.04)^{b}$ & $7.4^{b}$ & $4.0^{b}$ & 12.5 & $14.5^{b}$ \\
\hline Role emotional & $10.3(5.03)^{b}$ & $4.0(5.80)$ & $6.3^{b}$ & $6.8^{b}$ & -5.0 & $18.1^{b}$ \\
\hline Mental health & $4.6(2.13)^{b}$ & $5.1(2.30)^{b}$ & $4.6^{b}$ & $2.4^{b}$ & 2.8 & 5.0 \\
\hline
\end{tabular}

${ }^{a}$ Fabry Registry data are stratified by gender because Fabry disease is an X-linked disorder, and men are generally more severely affected. Data in parentheses represent standard error of the mean.

For agalsidase beta data, ${ }^{b} P<0.05$ by repeated measures models with adjustment for age at initiation of agalsidase beta treatment. For abatacept data, ${ }^{b} P<0.01$ by analysis of variance. ${ }^{27}$ For natalizumab data, ${ }^{b} P<0.05$ by analysis of covariance corrected for differences in baseline scores. ${ }^{28}$ For pregabalin data, ${ }^{b} P<0.01$ by analysis of covariance corrected for differences in baseline scores. ${ }^{29}$ For imiglucerase data, ${ }^{b} P<0.05$ by Wilcoxon signed-rank test. ${ }^{30}$

treatment. Indeed, when patients were grouped according to baseline SF-36 scores, those with baseline scores less than the median exhibited much larger improvements in HRQL, among both genders. However, this latter finding could also reflect a methodological ceiling effect; that is, patients with scores in the higher range (i.e., "better" scores) have less room for improvement because of the fact that the scale stops at 100 . In this population, $55 \%$ of women $(34 / 59)$ and $61 \%$ of men $(43 / 71)$ reported a maximum score in one or more of the eight scales (data not shown).

Age at initiation of treatment was an important factor in men but not in women. Men who were aged 33 years or older (the median age at which agalsidase beta treatment was initiated) when they began receiving treatment showed greater improvements in SF-36 scores and improved in more scales than did men who were younger than 33 years when treatment was initiated. The older group of men also had lower SF-36 scores in all eight scales at baseline. As described earlier, this provided more room to improve in the 100-point scale, when compared with younger men. However, women aged 48 years or older (the median age at which agalsidase beta treatment was initiated in women) had statistically significant responses in the same number of subscales as did younger women. Unlike in men, older women did not have lower baseline SF-36 scores, when compared with the younger group.

The magnitude of these improvements in HRQL was put into some perspective by comparing these changes with those reported by patients undergoing treatment for other chronic diseases. In previous studies, HRQL in untreated men with Fabry disease has been reported to be similar to that of patients undergoing renal dialysis and patients affected with acquired immune deficiency syndrome. ${ }^{12} \mathrm{HRQL}$ in untreated women with Fabry disease has been reported to be similar to that of patients with rheumatoid arthritis or multiple sclerosis. ${ }^{14}$ The improvement in HRQL reported by patients with Fabry disease in response to agalsidase beta treatment is substantial; after 12-24 months, men reported average improvements of 9 or more points in five of the eight 100-point scales. Much smaller improvements were reported by patients with rheumatoid arthritis who were treated with abatacept,${ }^{27}$ patients with multiple sclerosis who were treated with natalizumab, ${ }^{28}$ and patients with central neuropathic pain who were treated with pregabalin. ${ }^{29}$ In particular, patients with Fabry disease reported substantially greater improvements in the Physical Functioning and Role Physical scales after agalsidase beta treatment, compared with the effects of natalizumab in patients with multiple sclerosis or 
pregabalin in patients with central neuropathic pain. This is relevant because patients with Fabry disease frequently experience chronic fatigue, as do patients with multiple sclerosis or neuropathic pain. Among the four diseases that were compared with Fabry disease, the overall improvement in the HRQL of Fabry men in response to agalsidase beta most closely resembled that of patients with type 1 Gaucher disease who were treated with imiglucerase. ${ }^{30}$ Although type 1 Gaucher disease affects a different set of organ systems than Fabry disease (primarily liver, spleen, and bone marrow), it is also a lysosomal storage disorder that is associated with chronic pain and longterm disability. ${ }^{33}$ Nevertheless, these comparisons are associated with certain caveats. Rheumatoid arthritis and multiple sclerosis most commonly affect women, whereas the most severely affected patients with Fabry disease are men. In addition, not all patients with neuropathic pain respond to pregabalin, and a relatively small number of patients $(N=32)$ were included in the study of imiglucerase treatment on Gaucher disease, ${ }^{30}$ which, similar to Fabry disease, is a very rare disorder.

There are several limitations that must be considered when interpreting these analyses. The findings are limited by the voluntary and observational nature of data reported to registries. The Fabry Registry recommends that physicians ask patients who receive agalsidase beta treatment to complete the SF-36 health survey on enrollment, at baseline (i.e., immediately before initiation of treatment), and at 6-month intervals thereafter. However, not all patients visit their physicians at the recommended intervals, and treating physicians determine the actual frequency at which the various assessments are administered. Relatively, few patients who have been treated with agalsidase beta have reported SF-36 assessments at each recommended time point. Compared with other agalsidase beta-treated men in the Fabry Registry, 71 men who met the criteria to be included in the HRQL cohort were diagnosed at a younger age, initiated treatment at a younger age, and had experienced fewer serious clinical events at the time they began treatment. This suggests that these men were not in a more advanced state of disease progression than other men in the Fabry Registry at the time they began agalsidase beta treatment. There were no significant differences in these parameters between the 59 women included in the HRQL cohort compared with other agalsidase betatreated women in the Fabry Registry.

The second limitation to consider is that the SF-36 measures relatively generic aspects of HRQL. Generally, generic instruments are less sensitive to changes compared with specific instruments, and there are likely to be certain aspects of HRQL that affect patients with Fabry disease but are not measured by the SF-36. For example, it has previously been shown that $62 \%$ of men with Fabry disease experienced impact on their sex life ${ }^{34}$; an aspect not measured by the SF-36. The same study reported that $70 \%$ experienced interference with their job and $62 \%$ were limited in social activities, which is consistent with the findings from the current analyses.

Perhaps, the most important limitation of these findings is the lack of a parallel control group. It is difficult to identify untreated Fabry Registry patients to serve as an appropriate comparator group for this population. Patients with Fabry disease who receive treatment are generally more severely affected by Fabry disease than those who have not been treated. In addition, HRQL declines with age in untreated patients with Fabry disease, particularly women. ${ }^{8}$ Therefore, untreated patients must be matched by age and gender with treated patients. At this point, there are too few agalsidase beta-treated patients in the Fabry Registry who have appropriate longitudinal SF-36 data to subgroup patients in this manner and perform meaningful sta- tistical analyses. For this reason, the effect of agalsidase beta treatment on HRQL was expressed as average change from baseline, such that each patient's baseline data served as his or her own pretreatment control.

Another important issue is that pain and quality of life are subjective experiences that can be strongly influenced by patients' expectations. ${ }^{32,35}$ A Phase 3 randomized double-blinded placebocontrolled study showed that agalsidase beta was a safe and effective treatment for Fabry disease. ${ }^{15}$ After 20 weeks of treatment, patients treated with agalsidase beta exhibited significant clearance of globotriaosylceramide from the microvascular endothelium of various tissues, whereas placebo-treated patients did not. ${ }^{15}$ However, both the agalsidase beta-treated and the placebo-treated patients reported significant decreases in pain and significant improvements in the Role Physical component of the SF-36 after 20 weeks, ${ }^{15}$ which made it impossible to differentiate treatment-related effects on pain and HRQL from a placebo effect. These patients were followed in a long-term extension study in which all patients received agalsidase beta for up to 54 months. ${ }^{17}$ Renal function remained stable after 54 months of treatment, and significant improvements in the Physical Functioning, Body Pain, and Role Emotional SF-36 scales were also reported at that time. ${ }^{17}$ The improvements in HRQL reported after these longer durations of treatment (54 months in the Phase 3 extension study and 24-36 months in the current Fabry Registry analyses) may be more likely to be treatment-related effects of agalsidase beta than the improvements reported after the initial 20 weeks of the Phase 3 study. ${ }^{15}$

In conclusion, our analyses indicate that long-term treatment with agalsidase beta is associated with substantially improved HRQL in both men and women with Fabry disease; the effect is more pronounced in men. In men, largest improvements were in the impact of physical and mental health on performance of work and daily activities. Women also reported the largest improvements in the impact of physical health not only on performance but on general health perception and social function. Lower HRQL before beginning treatment was associated with greater improvement in response to agalsidase beta, as was older age among men, but not women. All patients with Fabry disease should be monitored closely and appropriately treated for pain and other symptoms that limit their everyday activities.

\section{ACKNOWLEDGMENTS}

The authors thank many patients who have agreed to participate in the Fabry Registry and the physicians and research coordinators who have entered clinical data on these patients.

\section{REFERENCES}

1. Desnick RJ, Ioannou YA, Eng CM. $\alpha$-Galactosidase A deficiency: Fabry disease. In: Scriver CR, Beaudet AL, Sly WS, Valle DV, editors. The metabolic and molecular bases of inherited disease, 8th ed. New York: McGraw Hill, 2001:3733-3774.

2. Ries M, Ramaswami U, Parini R, et al. The early clinical phenotype of Fabry disease: a study on 35 European children and adolescents. Eur J Pediatr 2003;62:767-772.

3. Ries M, Gupta S, Moore DF, et al. Pediatric Fabry disease. Pediatrics 2005; 115:e344-e355.

4. Hopkin RJ, Bissler J, Banikazemi M, et al. Characterization of Fabry disease in 352 pediatric patients in the Fabry Registry. Pediatr Res 2008;64:550 555

5. Branton MH, Schiffmann R, Sabnis SG, et al. Natural history of Fabry renal disease: influence of alpha-galactosidase A activity and genetic mutations on clinical course. Medicine 2002;81:122-138.

6. Mehta A, Ricci R, Widmer U, et al. Fabry disease defined: baseline clinical manifestations of 366 patients in the Fabry Outcome Survey. Eur J Clin Invest 2004;34:236-242.

7. Ortiz A, Oliveira JP, Waldek S, et al. Nephropathy in males and females with Fabry disease: cross-sectional description of patients before treatment with enzyme replacement therapy. Nephrol Dial Transplant 2008;23:1600-1607. 
8. Wilcox WR, Oliveira JP, Hopkin RJ, et al. Females with Fabry disease frequently have major organ involvement: lessons from the Fabry Registry. Mol Genet Metab 2008;93:112-128.

9. Sims K, Politei J, Banikazemi M, et al. Stroke in Fabry disease frequently occurs before diagnosis and in the absence of other clinical events: natural history data from the Fabry Registry. Stroke 2009;40:788-794.

10. Waldek S, Patel M, Banikazemi M, et al. Life expectancy and cause of death in males and females with Fabry disease: findings from the Fabry Registry. Genet Med 2009;11:790-796.

11. Cole AL, Lee PJ, Hughes DA, et al. Depression in adults with Fabry disease: a common and under-diagnosed problem. J Inherit Metab Dis 2007;30:943951.

12. Gold KF, Pastores GM, Botteman MF, et al. Quality of life of patients with Fabry disease. Qual Life Res 2002;11:317-327.

13. Miners AH, Holmes A, Sherr L, et al. Assessment of health-related qualityof-life in males with Anderson Fabry disease before therapeutic intervention. Qual Life Res 2002;11:127-133.

14. Street NJ, Yi MS, Bailey LA, et al. Comparison of health-related quality of life between heterozygous women with Fabry disease, a healthy control population, and patients with other chronic disease. Genet Med 2006;8:346-353.

15. Eng CM, Guffon N, Wilcox WR, et al. Safety and efficacy of recombinant human alpha-galactosidase A-replacement therapy in Fabry's disease. N Engl J Med 2001;345:9-16.

16. Wilcox WR, Banikazemi M, Guffon N, et al. Long-term safety and efficacy of enzyme replacement therapy for Fabry disease. Am J Hum Genet 2004; 75:65-74.

17. Germain DP, Waldek S, Banikazemi M, et al. Sustained, long-term renal stabilization after 54 months of agalsidase beta therapy in patients with Fabry disease. J Am Soc Nephrol 2007;18:1547-1557.

18. Banikazemi M, Bultas J, Waldek S, et al. Agalsidase-beta therapy for advanced Fabry disease. Ann Intern Med 2007;146:77-86.

19. Weidemann F, Niemann M, Breunig F, et al. Long-term effects of enzyme replacement therapy on Fabry cardiomyopathy: evidence for a better outcome with early treatment. Circulation 2009;119:524-529.

20. Hoffmann B, Garcia de Lorenzo A, Mehta A, et al. Effects of enzyme replacement therapy on pain and health related quality of life in patients with Fabry disease: data from FOS (Fabry Outcome Survey). J Med Genet 2005;42:247-252.

21. Mehta A, Beck M, Elliott P, et al. Enzyme replacement therapy with agalsidase alfa in patients with Fabry's disease: an analysis of registry data. Lancet 2009;374:1986-1996.

22. Eng CM, Germain DP, Banikazemi M, et al. Fabry disease: guidelines for the evaluation and management of multi-organ system involvement. Genet Med 2006;8:539-548.

23. Ware JE, Snow KK, Kosinski M, et al. SF-36 ${ }^{\circledR}$ Health Survey manual and interpretation guide. Boston, MA: New England Medical Center, the Health Institute, 1993

24. Ware JE. SF-36 health survey update. Spine 2000;25:3130-3139.

25. Angst F, Aeschlimann A, Stucki G. Smallest detectable and minimal clinically important differences of rehabilitation intervention with their implications for required sample sizes using WOMAC and SF-36 quality of life measurement instruments in patients with osteoarthritis of the lower extremities. Arthritis Rheum 2001;45:384-391.

26. Bjorner JB, Wallenstein GV, Martin MC, et al. Interpreting score differences in the SF-36 Vitality scale: using clinical conditions and functional outcomes to define the minimally important difference. Curr Med Res Opin 2007;23: 731-739.

27. Westhovens R, Cole JC, Li T, et al. Improved health-related quality of life for rheumatoid arthritis patients treated with abatacept who have inadequate response to anti-TNF therapy in a double-blind, placebo-controlled, multicentre randomized clinical trial. Rheumatology 2006;45:1238-1246.

28. Rudick RA, Miller D, Hass S, et al. Health-related quality of life in multiple sclerosis: effects of natalizumab. Ann Neurol 2007;62:335-346.

29. Vranken JH, Dijkgraaf MG, Kruis MR, et al. Pregabalin in patients with central neuropathic pain: a randomized, double-blind, placebo-controlled trial of a flexible-dose regimen. Pain 2008;136:150-157.

30. Weinreb N, Barranger J, Packman S, et al. Imiglucerase (Cerezyme ${ }^{\circledR}$ ) improves quality of life in patients with skeletal manifestations of Gaucher disease. Clin Genet 2007;71:576-588.

31. Kosinski M, Zhao SZ, Dedhiya S, et al. Determining minimally important changes in generic and disease-specific health-related quality of life questionnaires in clinical trials of rheumatoid arthritis. Arthritis Rheum 2000;43: $1478-1487$

32. Schwartz CE, Bode R, Repucci N, et al. The clinical significance of adaptation to changing health: a meta-analysis of response shift. Qual Life Res 2006;15:1533-1550.

33. Beutler F, Grabowski GA. Gaucher disease. In: Scriver CR, Beaudet AL, Sly WS, Valle DV, editors. The metabolic and molecular bases of inherited disease, 8th ed. New York: McGraw Hill, 2001:3635-3668.

34. MacDermot KD, Holmes A, Miners AH. Anderson-Fabry disease: clinica manifestations and impact of disease in a cohort of 98 hemizygous males. $J$ Med Genet 2001;38:750-760.

35. Turner JA, Deyo RA, Loeser JD, et al. The importance of placebo effects in pain treatment and research. JAMA 1994;271:1609-1614. 